KCL-MTH-00-06

hep-th/0002218n

Feb 25, 2000

\title{
On the boundary Ising model with disorder operators
}

\author{
G.M.T. Watts" \\ Department of Mathematics, King's College London, \\ Strand, London, WC2R 2LS, U.K.
}

\begin{abstract}
We extend the well-known method of calculating bulk correlation functions of the conformal Ising model via bosonisation to situations with boundaries. Oshikawa and Affleck have found the boundary states of two decoupled Ising models in terms of the orbifold of a single free boson compactified on a circle of radius $r=1$; we adapt their results to include disorder operators. Using these boundary states we calculate the expectation value of a single disorder field on a cylinder with free boundary conditions and show that in the appropriate limits we recover the standard and frustrated partition functions. We also show how to calculate Ising correlation functions on the upper half plane.
\end{abstract}

\footnotetext{
1e-mail: gmtw@mth.kcl.ac.uk
} 


\section{Introduction}

There is a well-known construction of correlation functions in two decoupled critical Ising models as correlation functions in a $c=1$ free boson theory [6, 7]. A natural question to ask is how to extend this to the boundary model and how to extend the analysis of Cardy and Lewellen [2,3] to include non-local bulk fields such as the Ising disorder field.

We first recall the Ising model field content. The maximal set of primary fields one normally considers are the identity field $\mathbb{1}$ of weight $(0,0)$; the spin field $\sigma$ and disorder field $\mu$ of weights $(1 / 16,1 / 16)$; the energy operator $\epsilon$ of weight $(1 / 2,1 / 2)$; and the fermion fields $\psi$ and $\bar{\psi}$ of weights $(1 / 2,0)$ and $(0,1 / 2)$ respectively. These fields are not mutually local, and the three maximal sets of mutually local fields are

$$
\{\mathbb{1}, \sigma, \epsilon\},\{\mathbb{1}, \mu, \epsilon\},\{\mathbb{1}, \psi, \bar{\psi}, \epsilon\} .
$$

We shall take the point of view that our primary fields are the local set $\mathbb{1}, \sigma$ and $\epsilon$ and we can then add the disorder field $\mu$ at the expense of introducing disorder lines joining pairs of disorder fields. The effect of the disorder lines is that the correlation function changes sign when a spin field passes through a disorder line; without these disorder lines the correlation functions containing both spin and disorder fields would be double-valued. We also recall that bulk correlation functions are related by duality, under which $\sigma \leftrightarrow \mu, \epsilon \leftrightarrow-\epsilon$.

A conformally-invariant boundary condition on a conformal field theory the upper half plane defines a set of correlation functions of the bulk fields satisfying $T(x)=\bar{T}(x)$ on the $x$-axis. The boundary theory of the local field theory containing $\sigma$ and $\epsilon$ was investigated by Cardy [2] who found that (under certain assumptions such as the uniqueness of the vacuum) there are three such boundary conditions, denoted by ' + ', '-' and ' $f$ ', which have the interpretation that the spin variable in the lattice realisation is fixed up, fixed down and free, respectively.

The fields on the boundary can be classified into primary and descendent fields. Under Cardy's assumptions, one finds that the identity is the only primary field on the \pm boundaries, whereas on the free b.c. there is a non-trivial primary field $\sigma^{B}(x)$ of weight $1 / 2$ which has the interpretation of the boundary spin field. One can further consider fields $\psi^{(\alpha \beta)}$ which interpolate different b.c.'s $\alpha$ and $\beta$, and which again fall into primary and descendent fields; $\psi^{( \pm \mp)}$ have weight $1 / 2$ and $\psi^{( \pm f)}, \psi^{(f \pm)}$ have weight $1 / 16$.

To complete the picture one needs the structure constants in the bulk-boundary opes (expressing the expansion of a bulk field in boundary fields) and the opes of boundary fields. For the Ising model these were found by Cardy and Lewellen [3], and the bulk-boundary opes take the form

$$
\left.\begin{aligned}
\left.\sigma(z, \bar{z})\right|_{ \pm} & \sim{ }^{( \pm)} B_{\sigma}^{1} \cdot|2 y|^{-1 / 8}
\end{aligned} \quad \epsilon(z, \bar{z})\right|_{\alpha} \sim{ }^{(\alpha)} B_{\epsilon}^{1} \cdot|2 y|^{-1}
$$

where ${ }^{( \pm)} B_{\sigma}^{1}= \pm 2^{1 / 4},{ }^{(f)} B_{\sigma}^{\sigma^{B}}=2^{-1 / 4},{ }^{( \pm)} B_{\epsilon}^{1}=1,{ }^{(f)} B_{\epsilon}^{1}=-1$.

A natural question is how to include the bulk disorder field in this analysis. Roughly speaking, fixed and free b.c.'s are interchanged under duality. However, as noted in [州, this leads to an apparent contradiction between duality and the (conjectured) $g$-theorem for boundary 
renormalisation group flows. This conjecture states that the boundary entropy $g$ in a unitary theory decreases along a renormalisation group flow [1]. The values for the free and fixed b.c.'s are $g_{\text {free }}=1, g_{\text {fixed }}=2^{-1 / 2}$. When the free b.c.'s are perturbed by a boundary magnetic field, there is a flow to the fixed b.c. However, under duality there should be a similar flow from fixed to free induced by the boundary disorder field, which would apparently be forbidden by the $g$-theorem. A resolution of this contradiction was obtained in [0] in which it was shown that a careful consideration of the two local field theories leads one to the result that for the local theory containing $\{\mathbb{1}, \mu, \epsilon\}$ one has three different boundary conditions, 'fixed', 'free+' and 'free-' which are dual to 'free', 'fixed+' and 'fixed-'. In terms of these new b.c.'s, the flow is from 'fixed' with $g=1$ to 'fixed \pm ' with $g=2^{-1 / 2}$, in agreement with the $g$-theorem. Here we suggest another resolution adapted to our situation in which we wish to remain within a theory containg the spin field. As we see below, the boundary disorder field is locally identical to the operator interpolating fixed + and fixed - b.c.'s, and so one can formulate a perturbation by the boundary disorder field only in a Hilbert space describing the direct sum of + and - b.c.'s. Since the actual flow is now from a direct sum of + and - b.c.'s with $g=2 \cdot 2^{-1 / 2}=2^{1 / 2}$ to free with $g=1, g$ decreases along this flow, and again there is no contradiction with the $g$-theorem.

Using duality, the bulk-boundary ope of the disorder field in the free b.c. should be dual to that of the spin field in fixed b.c.'s; i.e. in the free b.c. the bulk disorder field only couples to the identity,

$$
\left.\mu(z, \bar{z})\right|_{\text {free }} \sim^{(\text {free })} B_{\mu}^{1}|2 y|^{-1 / 8}, \quad{ }^{(\text {free })} B_{\mu}^{1}= \pm 2^{1 / 4},
$$

where the sign ambiguity reflects the overall ambiguity in correlation functions involving the disorder field.

However, when a disorder field approaches a free boundary, it carries its associated disorder line with it. Since the leading term in the ope of the disorder field with the boundary is the identity operator, this means that a disorder line can end on a free boundary at an operator of weight 0 . This explains the observation in [11] that the boundary state describing a circular boundary in the plane with free b.c. with a disorder line is rotationally invariant - one might at first sight think that the presence of the disorder line ending on the boundary would lead to a dependence on the position of the disorder line on the boundary, but since it couples to field of weight 0 this is not the case. Alternatively, the disorder line simply represents a cut in the value of correlation functions and has no physical content, it does not break the rotational symmetry of the correpsonding boundary state.

We shall see this directly in section 3 where we construct the one-point function of the bulk disorder field on the cylinder with free b.c.'s which interpolates the frustrated and unfrustrated partition functions as the disorder field crosses the cylinder.

In fixed b.c.'s conversely, the bulk disorder field will only couple to a boundary field of weight $1 / 2$, the boundary disorder field (also called the boundary freedom field 畞) $\mu^{B}(x)$,

$$
\left.\mu(z, \bar{z})\right|_{\text {fixed }} \sim^{\text {(fixed) }} B_{\mu}^{\mu^{B}}|2 y|^{3 / 8} \cdot \mu^{B}(x), \quad{ }^{(\text {fixed })} B_{\mu}^{\mu^{B}}=2^{-1 / 4},
$$

We have denoted the b.c. by 'fixed' rather than by ' \pm ' since the presence of the disorder fields means that it is not possible to unambiguously identify a fixed b.c. as either ' + ' or '-'. The ability to move a disorder line across a boundary at no cost (other than a sign change when 
it passes through a spin field) is also true for the fixed b.c., thereby changing the b.c. from + to - or vice versa. Using this property we see that the boundary disorder field on a fixed b.c. can be identified with a $( \pm \mp)$ boundary condition changing operator connected to a disorder line, as shown in figure 1

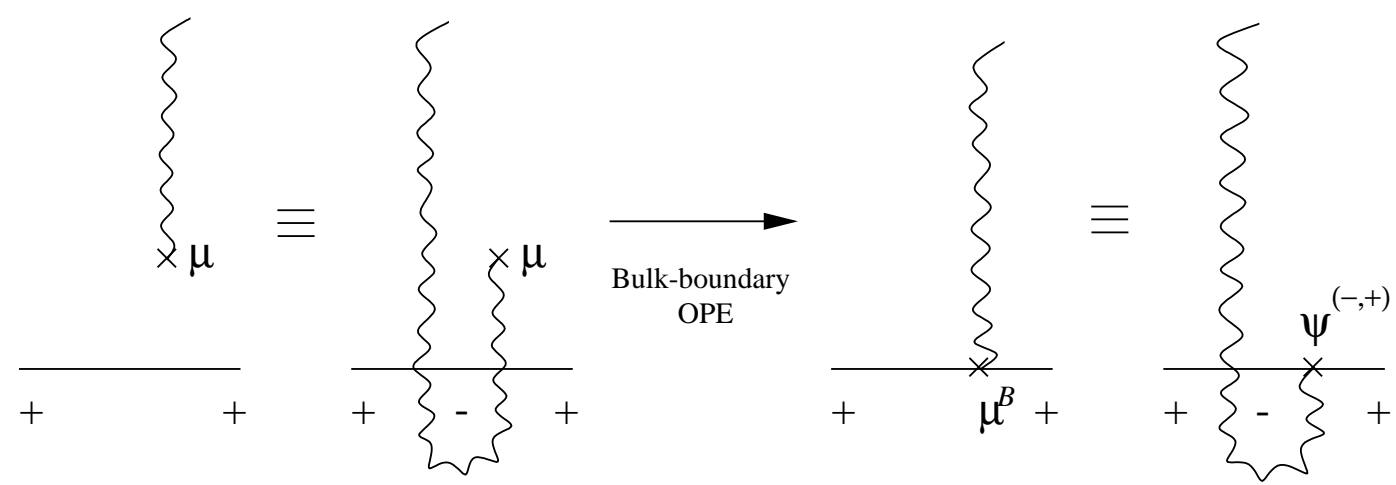

Figure 1: the equivalence of the boundary disorder field and the boundary changing operator

One can 'pull' the disorder line attached to the bulk disorder field down and to the left until it crosses the edge of the upper-half-plane leaving the bulk disorder field attached to a disorder line that ends directly on the boundary at a field of weight 0 . When the bulk field then approaches the boundary, this disorder line shrinks and locally the leading term in the bulk-boundary ope is the boundary-condition-changing operator interpolating \pm b.c.'s. This shows that the boundary disorder field is locally identical to a boundary-condition changing operator, the only difference being the position of disorder lines.

\section{Bosonisation formulae}

As explained in [9], one can realise two independent copies of the Ising model with local fields $\{1, \sigma, \epsilon\}$ as the $\mathbb{Z}_{2}$ orbifold of a single free boson compactified on a circle of radius $r=1$. If we label the fields in the two Ising models by 1 and 2 , this is generated by

$$
\left\{\mathbb{1}, \sigma_{1}, \epsilon_{1}, \sigma_{2}, \epsilon_{2}\right\}
$$

and is a local field theory; we shall call this model A. One can consider a different $c=1$ theory containing (amongst others) the following alternative mutually local set of fields,

$$
\left\{\mathbb{1}, \sigma_{1} \sigma_{2}, \mu_{1} \mu_{2}, \epsilon_{1}+\epsilon_{2}, \epsilon_{1} \epsilon_{2}\right\},
$$

which we shall call model B. This is the $\mathbb{Z}_{2}$ orbifold of model A, i.e. the un-orbifolded free boson and contains only symmetric combinations of the fields in the two Ising models, although not all - for example it does not contain $\sigma_{1}+\sigma_{2}$ which would be non-local with respect to $\mu_{1} \mu_{2}$.

The advantage of considering model $\mathrm{B}$ is that it enables one to construct correlation functions $\langle\mathcal{O}\rangle$ in a single Ising model as the square root of the correlation function $\left\langle\mathcal{O}_{1} \mathcal{O}_{2}\right\rangle$ in a free boson theory, as explained in [7]. The correlation functions in model B are single-valued, but 
taking the square-root leads to the (possibly) multi-valued correlation functions in the Ising model.

The bosonisation formulae on the plane are [7]

$$
\begin{aligned}
\sigma_{1} \sigma_{2} & =\sqrt{2}: \cos \frac{1}{2} \varphi:, & \mu_{1} \mu_{2} & =\sqrt{2}: \sin \frac{1}{2} \varphi:, \\
\epsilon_{1} \epsilon_{2} & =-: \partial \varphi \bar{\partial} \varphi:, & \epsilon_{1}(z, \bar{z})+\epsilon_{2}(z, \bar{z}) & =2: \cos \varphi:,
\end{aligned}
$$

Note that the field representing $\mu_{1} \mu_{2}$ is absent from the orbifold model of [10].

We now consider this model on a cylinder of length $L$, circumference $R$, which we take to be the rectangle in the complex $z$ plane with vertices $0, R, i L, R+i L$. This can be mapped to an annulus in the $\zeta$ plane by $\zeta=\exp (-2 \pi i z / R)$. In this form, the boundary conditions on the two ends of the cylinder are represented by boundary states in the full bulk theory.

All the possible boundary states for the model A were found by Oshikawa and Affleck in [10]. To use their results, we first give our conventions. We take the mode expansion of $\varphi(\zeta, \bar{\zeta})$ on the plane to be

$$
\varphi(\zeta, \bar{\zeta})=q-i p \ln (\zeta \bar{\zeta})-i w \ln (\zeta / \bar{\zeta})+i \sum_{n \neq 0}\left(\frac{a_{n}}{n} \zeta^{-n}+\frac{\bar{a}_{n}}{n} \bar{\zeta}^{-n}\right)
$$

where $w$ is the winding number, $[q, p]=i$ and $\left[a_{m}, a_{n}\right]=\left[\bar{a}_{m}, \bar{a}_{n}\right]=m \delta_{m,-n}$. With this choice of $\varphi$, compactification on a circle of radius 1 in the sense of [9] means we identify $\varphi \cong \varphi+4 \pi$, and hence $w$ takes integer and $p$ half-integer values (n.b. this is different to [6]). For the boundary states we consider, we only need the Dirichlet-type Ishibashi states of zero winding number, viz.

$$
|k\rangle\rangle=\exp \left(\sum_{n>0} \frac{a_{-n} \bar{a}_{-n}}{n}\right)|k\rangle
$$

which satisfy

$$
\left.\left(a_{n}-\bar{a}_{-n}\right)|k\rangle\right\rangle=0 .
$$

The boundary states in the orbifold model also contain states in the twisted sector, but these will play no role for us.

Although the space of states in models A and B differ, one of the boundary conditions of [10] is common to the two models, and that is the condition that the spins on both models are 'free'. We denote this by $\mid$ free $\rangle$ and it is given by

$$
\left.\mid \text { free }\rangle=\sum_{k \in \mathbb{Z}}(-1)^{k}|k\rangle\right\rangle \text {. }
$$

To check that this does indeed correspond to free boundary conditions, one can calculate the partition function for the system with this boundary state on the two ends. Given the cylinder Hamiltonian

$$
H(R)=\frac{2 \pi}{R}\left(L_{0}+\bar{L}_{0}-1 / 12\right)
$$

the partition function with this 'free' boundary condition on the both ends of the cylinder is

$$
\begin{aligned}
Z_{\text {free,free }} & =\left\langle\text { free }\left|e^{-L H(R)}\right| \text { free }\right\rangle=\sum_{k \in \mathbb{Z}} \tilde{q}^{k^{2} / 2} / \eta(\tilde{\tau})=\theta_{3}(0 \mid \tilde{\tau}) / \eta(\tilde{\tau})=\theta_{3}(0 \mid \tau) / \eta(\tau) \\
& =\left(\chi_{0}(q)+\chi_{1 / 2}(q)\right)^{2}=\left(Z_{\text {free,free }}^{\text {Ising }}\right)^{2}
\end{aligned}
$$


where $\tilde{q}=\exp (-4 \pi L / R), q=\exp (-\pi R / L), \tilde{\tau}=2 i L / R, \tau=i R /(2 L), \chi_{h}(q)$ is the character of the $c=1 / 2$ Virasoro algebra representation of weight $h$, and the definitions and properties of the $\theta$ functions can be found in e.g. chap. 10 of $[6]$.

Hence the state |free $\rangle$ naturally describes free boundary conditions with no disorder lines (or an even number of disorder lines) ending on the boundary. To find the boundary state $\mid$ free $\left.^{\prime}\right\rangle$ for the system with an odd number of disorder lines, we can use the fact that a bulk disorder operator carries with it a disorder line and has the bulk-boundary ope

$$
\left.\mu(x+i y)\right|_{\text {free }} \sim 2^{1 / 4}(2 y)^{-1 / 8} \mathbb{1}+\ldots .
$$

Hence we can calculate $\mid$ free $\left.{ }^{\prime}\right\rangle$ by acting with the bosonic expression for $\mu_{1} \mu_{2}$ on $\mid$ free $\rangle$.

Using the standard expression for the normal ordered vertex operator on the cylinder expressed in terms of the field on the plane,

$$
: e^{i \alpha \varphi_{\text {cyl. }}(z, \bar{z})}:=\left(\frac{2 \pi}{R}\right)^{\alpha^{2}}|\zeta|^{\alpha^{2}} e^{i \alpha \varphi<(\zeta, \bar{\zeta})} e^{i \alpha q}|\zeta|^{2 \alpha p} e^{i \alpha \varphi>(\zeta, \bar{\zeta})}
$$

where $\zeta=\exp (-2 \pi i z / R)$ and

$$
\varphi>(\zeta, \bar{\zeta})=i \sum_{n}>0\left(\frac{a_{n}}{n} \zeta^{-n}+\frac{\bar{a}_{n}}{n} \bar{\zeta}^{-n}\right)
$$

one easily finds that

$$
\left.\left.: e^{i \alpha \varphi_{\text {cyl. }}(z, \bar{z})}:|k\rangle\right\rangle=\left(\frac{R}{\pi} \sinh \frac{2 \pi y}{R}\right)^{-\alpha^{2}}|\zeta|^{2 \alpha p} e^{i \alpha\left(\varphi_{<}(\zeta, \bar{\zeta})-\varphi_{<}(1 / \zeta, 1 / \bar{\zeta})\right)}|k+\alpha\rangle\right\rangle .
$$

Therefore, using the bosonisation formulae (2.3), we find that

$$
\left.\left.\mu_{1} \mu_{2}(x+i y) \mid \text { free }\right\rangle=-i \sqrt{2}(2 y)^{-1 / 4} \sum_{k \in \mathbb{Z}}(-1)^{k}|k+1 / 2\rangle\right\rangle+O\left(y^{3 / 4}\right),
$$

and so the boundary state $\mid$ free $\left.^{\prime}\right\rangle$ of a free boundary condition with an odd number of disorder lines is given by

$$
\left.\left.\mid \text { free }^{\prime}\right\rangle=-i \sum_{k \in \mathbb{Z}}(-1)^{k}|k+1 / 2\rangle\right\rangle
$$

As a check we can calculate the partition function for the Ising model with a disorder line ending on each end,

$$
\begin{aligned}
Z_{\text {free }^{\prime}, \text { free }^{\prime}} & =\left\langle\text { free }^{\prime}\left|e^{-L H(R)}\right| \text { free }^{\prime}\right\rangle=\sum_{r \in \mathbb{Z}+1 / 2} \tilde{q}^{r^{2} / 2} / \eta(\tilde{\tau})=\theta_{2}(0 \mid \tilde{\tau}) / \eta(\tilde{\tau})=\theta_{4}(0 \mid \tau) / \eta(\tau) \\
& =\left(\chi_{0}(q)-\chi_{1 / 2}(q)\right)^{2}=\left(Z_{\text {free }^{\prime}, \text { free }^{\prime}}^{\text {Ising }}\right)^{2}
\end{aligned}
$$

i.e. the square of the frustrated partition function [11] as should be the case.

As a final check,

$$
Z_{\text {free,free }^{\prime}}=\left\langle\text { free }\left|e^{-L H(R)}\right| \text { free }^{\prime}\right\rangle=0,
$$

reflecting the fact that there are no configurations with a single disorder line ending on one end of the cylinder and no disorder fields. 
Having found the boundary states for the two Ising models in (free) and (free'), we must complete the discussion by considering the case of fixed boundary conditions. In model A, all possible combinations of up and down conditions on the two models are possible, and all such boundary states have been found in [10]. In model B however, the spin fields only appear in the symmetric combination $\left(\sigma_{1} \sigma_{2}\right)$, and so one can only possibly distinguish the relative signs of the two spins. This means that the possible boundary conditions in model B are 'fixed same' and 'fixed opposite', giving the relative signs of the two spins. If we denote the corresponding boundary states by $\mid$ same $\rangle$ and $\mid$ opp. $\rangle$, they are given in terms of Oshikawa's and Affleck's boundary states as

$$
\begin{aligned}
\mid \text { same }\rangle & \left.=\frac{|++\rangle+|--\rangle}{\sqrt{2}}=\sum_{k \in \mathbb{Z}}|k / 2\rangle\right\rangle \\
\mid \text { opp. }\rangle & \left.=\frac{|+-\rangle+|-+\rangle}{\sqrt{2}}=\sum_{k \in \mathbb{Z}}(-1)^{k}|k / 2\rangle\right\rangle
\end{aligned}
$$

Using (2.3) and (2.8) one finds that

$$
\begin{aligned}
\left.\sigma_{1} \sigma_{2}(x+i y) \mid \text { same }\right\rangle & \left.=+\sqrt{2}(2 y)^{-1 / 4} \mid \text { same }\right\rangle+O\left(y^{3 / 4}\right), \\
\left.\sigma_{1} \sigma_{2}(x+i y) \mid \text { opp. }\right\rangle & \left.=-\sqrt{2}(2 y)^{-1 / 4} \mid \text { opp. }\right\rangle+O\left(y^{3 / 4}\right) .
\end{aligned}
$$

As a final comment, we note how the fixed and free boundary states are related by duality. If $V \varphi V=-\varphi$, then duality $\varphi \mapsto \pi-\varphi$ is implemented by the operator $D=V e^{i \pi p}$. Hence we have

$$
\left.\left.\left.D|k\rangle\rangle=e^{i \pi k}|-k\rangle\right\rangle, \quad D \mid \text { free }\right\rangle=\frac{\mid \text { same }\rangle+\mid \text { opp. }\rangle}{\sqrt{2}}, \quad D \mid \text { free }^{\prime}\right\rangle=\frac{\mid \text { same }\rangle-\mid \text { opp. }\rangle}{\sqrt{2}} .
$$

\section{Cylinder correlation functions}

We can also use these bosonisation formulae and boundary states to calculate correlation functions of fields on the cylinder. One interesting case to consider is the expectation value of a single disorder field with free b.c.'s on the two sides of the cylinder.

To recall, the cylinder is given by the rectangle in the upper half $z$ plane with vertices $0, R, i L, R+i L$, with the vertical edges $\operatorname{Re}(z)=0, R$ identified. We must also choose on which end of the cylinder the disorder line attached to the bulk field ends. We choose it to end on the top edge $\operatorname{Im}(z)=L$.

Using standard free field techniques, we can explicitly evaluate

$$
\begin{aligned}
\left\langle\mu_{1} \mu_{2}(i y)\right\rangle & =\left\langle\text { free }^{\prime}\left|e^{-L H(R)}\left(\sqrt{2}: \sin \frac{1}{2} \varphi(i y):\right)\right| \text { free }\right\rangle \\
& =\sqrt{2} \frac{\theta_{3}(\tilde{\nu} / 2 \mid \tilde{\tau})}{\eta(\tilde{\tau})}\left[\frac{1}{i R} \frac{\theta_{1}^{\prime}(0 \mid \tilde{\tau})}{\theta_{1}(\tilde{\nu} \mid \tilde{\tau})}\right]^{1 / 4} \\
& =\sqrt{2} \frac{\theta_{3}(\nu / 2 \mid \tau)}{\eta(\tau)}\left[\frac{1}{2 L} \frac{\theta_{1}^{\prime}(0 \mid \tau)}{\theta_{1}(\nu \mid \tau)}\right]^{1 / 4},
\end{aligned}
$$

where $\nu=y / L$ and $\tilde{\nu}=-2 i y / R$. This gives the cylinder expectation value in the Ising model as 


$$
\langle\mu(i y)\rangle_{\text {free }^{\prime} \text {,free }}=2^{1 / 4} \sqrt{\frac{\theta_{3}\left(\frac{\nu}{2} \mid \tau\right)}{\eta(\tau)}}\left|\frac{\theta_{1}^{\prime}(0 \mid \tau)}{2 L \theta_{1}(\nu \mid \tau)}\right|^{1 / 8} .
$$

This can also be found as the appropriate combination of the two chiral blocks given in (12.109) of [6].

As the disorder field approaches the $x$-axis, $\nu \rightarrow 0$, we find

$$
\begin{aligned}
\langle\mu(i y)\rangle_{\text {free free }} & =2^{1 / 4}(2 \pi \nu)^{-1 / 8}\left(\sqrt{\frac{\theta_{3}(0 \mid \tau)}{\eta(\tau)}}+O(\nu)\right)=2^{1 / 4}(2 y)^{-1 / 8}\left(\chi_{0}+\chi_{1 / 2}\right)+\ldots \\
& =2^{1 / 4}(2 y)^{-1 / 8} Z_{\text {free,free }}^{\text {Ising }}+\ldots
\end{aligned}
$$

This is the exactly what we would have expected from the bulk-boundary ope of the disorder field (1.2) at a free boundary. Hence we see explicitly that as the disorder field approaches the $x$-axis the disorder line shrinks to zero.

However, if the disorder field approaches the other side, the disorder line gets stretched across the cylinder. As it approaches the boundary $\operatorname{Im}(z)=L$ the leading term in the bulk-boundary ope will again be the identity operator, so that the leading behaviour of the correlation function should be the frustrated partition function, i.e. the partition function including a disorder line,

$$
Z_{\text {free',free }}^{\text {Ising }}=\chi_{0}-\chi_{1 / 2} ;
$$

Using (3.2) it is easy to check that this is in fact the case. Putting $y=L-\tilde{y}, \tilde{y}=L v, \nu=1-v$, we find

$$
\begin{aligned}
\langle\mu(i(L-\tilde{y}))\rangle_{\text {free,free }} & =2^{1 / 4} \sqrt{\frac{\theta_{4}\left(\frac{v}{2} \mid \tau\right)}{\eta(\tau)}}\left|\frac{\theta_{1}^{\prime}(0 \mid \tau)}{-2 L \theta_{1}(-v \mid \tau)}\right|^{1 / 8} \\
& =2^{1 / 4}(2 L v)^{-1 / 8}\left(\sqrt{\frac{\theta_{4}(0 \mid \tau)}{\eta(\tau)}}+O(v)\right) \\
& =2^{1 / 4}(2 \tilde{y})^{-1 / 8}\left(\chi_{0}-\chi_{1 / 2}\right)+\ldots,
\end{aligned}
$$

as expected. The two limits and the way the frustration line is stretched across the cylinder are shown below in Figure 2 .

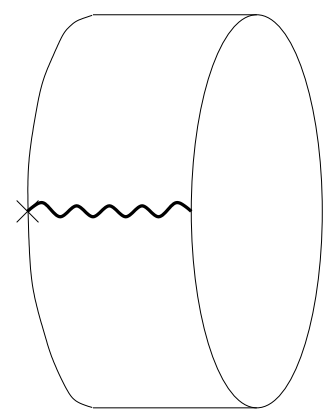

Frustrated partition function
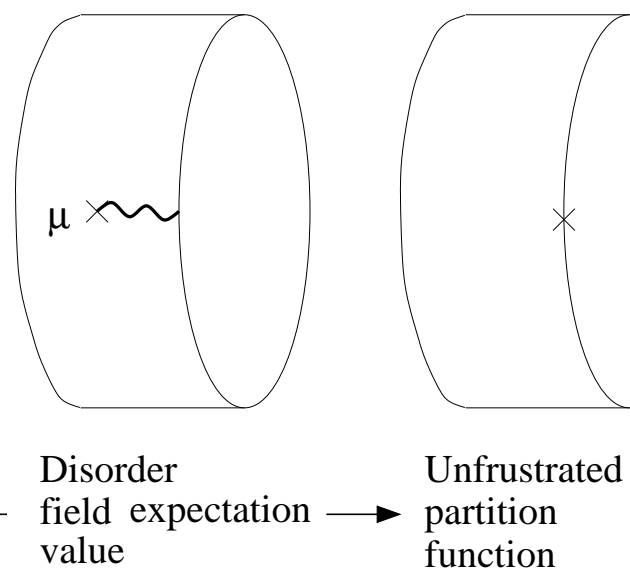

Figure 2. 


\section{Correlation functions on the upper half plane}

We finish with some comments on the structure of Ising correlation functions on the upper half plane (UHP). From [10], we can express the various boundary states in the B model in terms of various combinations of Dirichlet boundary states $\left|D\left(\varphi_{0}\right)\right\rangle$, i.e. states for which $\varphi$ takes the value $\varphi_{0}$ on the boundary:

$$
\begin{aligned}
\mid \text { free }\rangle & =\frac{|D(\pi)\rangle+|D(-\pi)\rangle}{\sqrt{2}} & \left.\mid \text { free }^{\prime}\right\rangle & =\frac{|D(\pi)\rangle-|D(-\pi)\rangle}{\sqrt{2}} \\
\mid \text { same }\rangle & =|D(0)\rangle & \mid \text { opp. }\rangle & =|D(2 \pi)\rangle
\end{aligned}
$$

Note that our field $\varphi$ is twice that of [10] and hence the values of $\varphi_{0}$ are also twice theirs.

It is easy to express a free boson $\varphi(\zeta, \bar{\zeta})$ satisfying $\varphi=\varphi_{0}$ on the $x$-axis in terms of a chiral boson $\phi(\zeta)$ as

$$
\varphi(\zeta, \bar{\zeta})=\varphi_{0}+\phi(\zeta)-\phi(\bar{\zeta})
$$

The only subtlety is that one cannot substitute this directly into vertex operators as the result will be ill-defined. We choose to define our normal ordering by

$$
: \exp (i \alpha \varphi(\zeta, \bar{\zeta})):\left.\right|_{D\left(\varphi_{0}\right)}=(2 \eta)^{-\alpha^{2}}: \exp \left(i \alpha\left(\varphi_{0}+\phi(\zeta)-\phi(\bar{\zeta})\right):\right.
$$

where $\zeta=\xi+i \eta$ with $\xi, \eta \in \mathbb{R}$.

If we also use the results of evaluating the bulk-boundary opes,

$$
\begin{aligned}
\sqrt{2}(2 \eta)^{-1 / 4}: \sin \frac{1}{2}(\phi(\zeta)-\phi(\bar{\zeta})): & =\frac{1}{\sqrt{2}}(2 \eta)^{3 / 4}[i \partial \phi(\xi)+O(\eta)] \\
\sqrt{2}(2 \eta)^{-1 / 4}: \cos \frac{1}{2}(\phi(\zeta)-\phi(\bar{\zeta})): & =\sqrt{2}(2 \eta)^{-1 / 4}\left[1+\frac{1}{2} \eta^{2}:(i \partial \phi(\xi))^{2}:+O\left(\eta^{3}\right)\right]
\end{aligned}
$$

we can further find the expressions for the boundary spin and disorder fields. We summarise the results in the following table of bosonisation formulae:

\begin{tabular}{cccc}
\hline & $D(0)$ & $D(\pi)$ & $D(-\pi)$ \\
\hline$\sigma_{1} \sigma_{2}$ & $\sqrt{2}(2 \eta)^{-\frac{1}{4}}: \cos \frac{\phi(\zeta)-\phi(\bar{\zeta})}{2}:$ & $-\sqrt{2}(2 \eta)^{-\frac{1}{4}}: \sin \frac{\phi(\zeta)-\phi(\bar{\zeta})}{2}:$ & $\sqrt{2}(2 \eta)^{-\frac{1}{4}}: \sin \frac{\phi(\zeta)-\phi(\bar{\zeta})}{2}:$ \\
$\mu_{1} \mu_{2}$ & $\sqrt{2}(2 \eta)^{-\frac{1}{4}}: \sin \frac{\phi(\zeta)-\phi(\bar{\zeta})}{2}:$ & $\sqrt{2}(2 \eta)^{-\frac{1}{4}}: \cos \frac{\phi(\zeta)-\phi(\bar{\zeta})}{2}:$ & $-\sqrt{2}(2 \eta)^{-\frac{1}{4}}: \cos \frac{\phi(\zeta)-\phi(\bar{\zeta})}{2}:$ \\
$\epsilon_{1} \epsilon_{2}$ & $: \partial \phi(\zeta)(\partial \phi)(\bar{\zeta}):$ & $: \partial \phi(\zeta)(\partial \phi)(\bar{\zeta}):$ & $: \partial \phi(\zeta)(\partial \phi)(\bar{\zeta}):$ \\
$\sigma_{1}^{B} \sigma_{2}^{B}$ & - & $-i \partial \phi(\xi)$ & $i \partial \phi(\xi)$ \\
$\mu_{1}^{B} \mu_{2}^{B}$ & $i \partial \phi(\xi)$ & - & - \\
\hline
\end{tabular}

Considering these formulae, we see that the only effect of taking the particular linear combinations in the free and free' b.c.'s is to ensure that correlators including odd and even numbers of disorder operators vanish respectively. As a result, we can safely evaluate correlators in the 'generic' free boundary conditions (i.e. not paying attention to the number of disorder lines ending on the boundary) by using simply the bosonisation formulae for $\varphi_{0}=\pi$. To summarise, we can calculate correlation functions in the UHP by using expressions for $D(0)$ to obtain fixed b.c.'s in the Ising models, and the expressions for $D(\pi)$ to obtain free b.c.'s. 
Note that as we have defined them, the bosonisation formulae for $D(0)$ and $D(\pi)$ are not exactly related by the duality $\varphi \mapsto \pi-\varphi$ which should simply interchange the expressions for the spin and disorder fields but instead differ by an irrelevant sign in the spin operator.

As an example we use these formulae to calculate a correlation function which would be hard to find using standard conformal field theory techniques, being equivalent to a five point chiral block. Consider the expectation value of a bulk spin field, a bulk disorder field and a boundary disorder field in fixed boundary conditions. Using the formulae for $D(0)$ we have

$$
\begin{aligned}
f(\zeta)= & \left(\left\langle\sigma(\zeta) \mu(i) \mu^{B}(1)\right\rangle_{\text {fixed }}\right)^{2}=\left\langle\sigma_{1} \sigma_{2}(\zeta) \mu_{1} \mu_{2}(i) \mu_{1}^{B} \mu_{2}^{B}(1)\right\rangle \\
= & 2(2 \eta)^{-1 / 4}(2)^{-1 / 4}\left\langle: \cos \left(\frac{1}{2}(\phi(\zeta)-\phi(\bar{\zeta}))\right):: \sin \left(\frac{1}{2}(\phi(i)-\phi(-i))\right): i \partial \phi(0)\right\rangle \\
= & -i 2^{-1 / 2} \eta^{-1 / 4} \\
& \left(\left(\frac{1}{\zeta}-\frac{1}{\bar{\zeta}}+\frac{1}{i}-\frac{1}{-i}\right)\left[\frac{(i-\zeta)(i+\bar{\zeta})}{(i-\bar{\zeta})(i+\zeta)}\right]^{1 / 4}-\left(\frac{1}{\zeta}-\frac{1}{\bar{\zeta}}-\frac{1}{i}+\frac{1}{-i}\right)\left[\frac{(i-\zeta)(i+\bar{\zeta})}{(i-\bar{\zeta})(i+\zeta)}\right]^{-1 / 4}\right)
\end{aligned}
$$

This expression is single valued in $\zeta$, but when we take the square root to find the result for a single Ising model there are square-root branch points at $\zeta=0$ and at $\zeta=i$. Where we put this branch cut has implications for the identification of the operators in the correlation function, as shown in figure 3 where the functions plotted are identical up to a choice of the position of the branch cut.

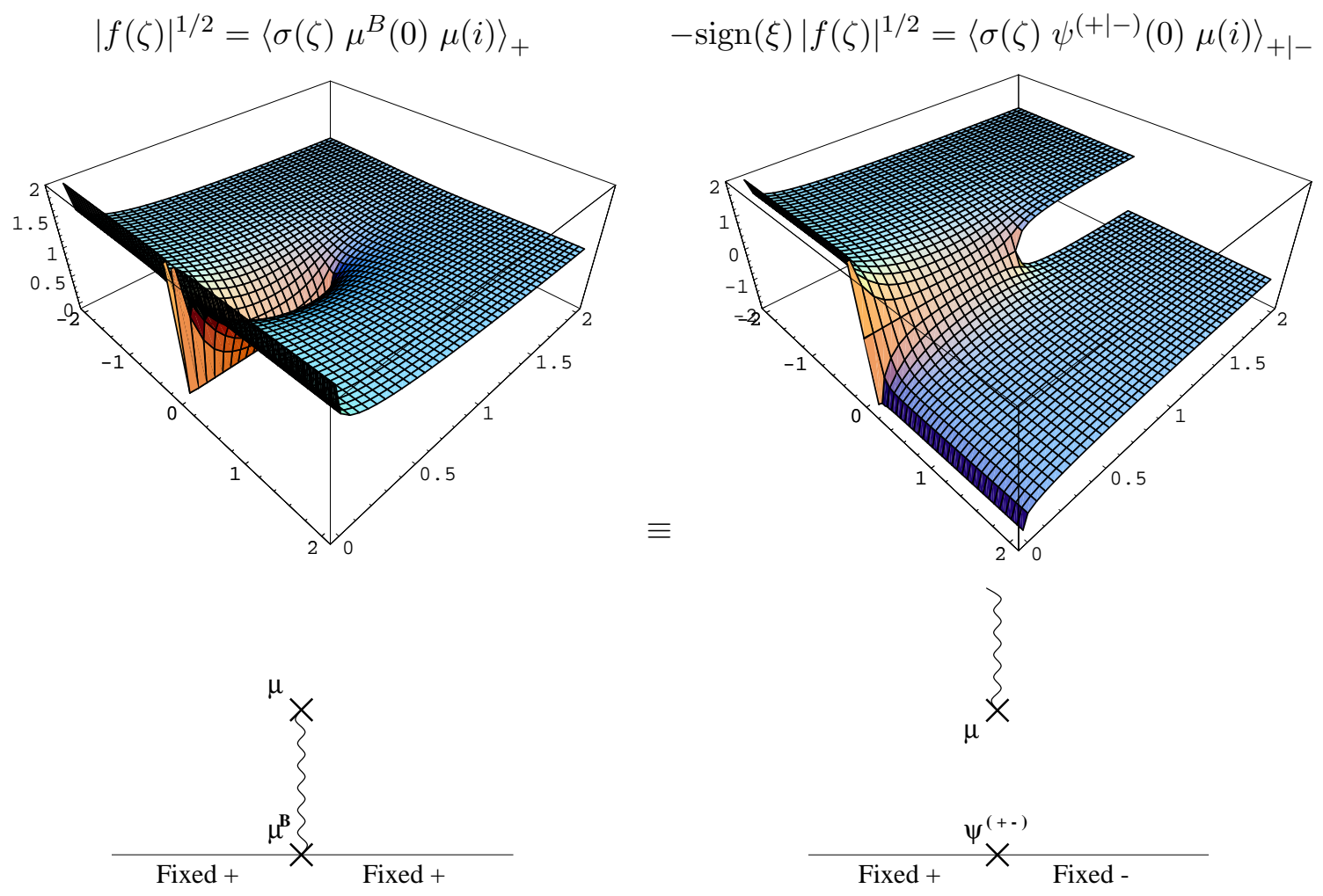

Figure 3: two choices of the position of the disorder line showing the equivalent interpretation of the boundary disorder field $\mu^{B}$ as the boundary-condition changing operator $\psi^{( \pm \mp)}$. 
In the first case we plot $|f|^{1 / 2}$, and with this choice the disorder line joins the bulk and boundary disorder fields, and the boundary condition appears to be uniform, fixed 'up'.

In the second case we plot $-\operatorname{sign}(\xi)|f|^{1 / 2}$, and the disorder line from the bulk spin field extends to infinity in the bulk, and the operator inserted at the origin appears to be a boundary changing operator interpolating fixed 'up' and fixed 'down' b.c.'s.

\section{Conclusions}

We have shown how the bulk bosonisation formulae of Di Francesco et al. [7] can be extended to the Ising model on the upper half plane when the bosonic field satisfies Dirichlet boundary conditions. This has helped understand the nature of boundary disorder fields, and has also helped clarify the results of [11] in which boundary states describing frustrated systems were found to be rotationally invariant - the result being that the disorder line ends on an operator of weight zero and hence is invariant under rotations.

The boundary conformal field theory we have been looking at is unusual on two counts. Firstly the bulk theory is non-local, and secondly it seems we are forced to consider boundary conditions with apparently two vacua; for the free case, these are vacua with even and odd numbers of disorder lines, and in the fixed these are the fixed up and fixed down vacua. Such boundary conditions fall outside Cardy's classification on both counts and it would be interesting to understand more generally the relations between the non-locality of the bulk theory and these peculiarities of the boundary theory.

The perturbation by the boundary disorder operator also provides a very simple example of a unitary perturbation by a boundary-condition changing operator. Given the renewed interest in such perturbations [ [ $]$, it will be of interest to generalise this to other models.

\section{Acknowledgments}

I would like to thank K. Graham, M.E. Ortiz, A. Recknagel, P. Ruelle and I. Runkel for many helpful discussions, and the organisers of the 1999 Oberwolfach meeting on "Mathematical Aspects of String Theory" for a very enjoyable meeting where this work was started. The work was supported in part by a TMR grant of the European Commission, contract reference ERBFMRXCT960012, and by an EPSRC advanced fellowship. 


\section{References}

[1] I. Affleck and A. W. W. Ludwig. Universal noninteger "ground-state degeneracy" in critical quantum systems, Phys. Rev. Lett. 67(2) (1991) 161-164.

[2] J. L. Cardy, Boundary conditions, fusion rules and the Verlinde formula, Nucl. Phys. B324 (1989) 581-596.

[3] J. L. Cardy and D. C. Lewellen, Bulk and boundary operators in conformal field theory, Phys. Lett. B259 No. 3 (1991) 274-278;

D. C. Lewellen, Sewing constraints for conformal field theories on surfaces with boundaries, Nucl. Phys. B372 (1992) 654-682.

[4] S. Carroll, M.E. Ortiz and W. Taylor, The Ising model with a boundary magnetic field on a random surface, Phys. Rev. Lett. 77 (1996) 3947-3950, hep-th/9605169; Boundary fields and renormalization group flow in the two-matrix model, Phys. Rev. D58 (1998) 046006, hep-th/9711008.

[5] S. Carroll, M.E. Ortiz and W. Taylor, Duality and reversal of renormalisation group flow, unpublished.

[6] P. Di Francesco, D. Sénéchal and P. Mathieu, Conformal Field Theory, Springer 1997

[7] P. Di Francesco, H. Saleur and J.-B. Zuber, Critical Ising correlation functions in the plane and on the torus, Nucl. Phys. 290 [FS20] (1987) 527-581.

[8] J. Fröhlich, O. Grandjean, A. Recknagel and V. Schomerus, Fundamental strings in Dp-Dq brane systems, hep-th/9912079.

[9] P. Ginsparg, Applied Conformal Field Theory, pp 1-168, in Fields, strings and critical phenomena, Les Houches 1988, eds E. Brézin and J. Zinn-Justin, pub. North Holland 1990.

[10] M. Oshikawa and I. Affleck, Defect Lines in the Ising Model and Boundary States on Orbifolds, Phys. Rev. Lett. 77 (1996) 2604-2607, hep-th/9606177; Boundary conformal field theory approach to the critical two-dimensional Ising model with a defect line, Nucl. Phys. B495 (1997) 533-582, cond-mat/9612187.

[11] P. Ruelle, Symmetric boundary conditions in boundary critical phenomena, J. Phys. A32 (1999) 8831-8850, hep-th/9904100. 